\title{
The Survey of Fishery Resources and Spatial Distribution Using DIDSON Imaging Sonar Data*
}

\author{
Wei Shen ${ }^{1}$, Long Yang ${ }^{2}$, Jin Zhang ${ }^{1, * *}$, and Guangxiong Peng ${ }^{3}$ \\ ${ }^{1}$ College of Marine Sciences, Shanghai Ocean University, Shanghai, 201306, China \\ j_zhang@shou.edu.cn \\ ${ }^{2}$ The First Institute of Oceanography, SOA, Qingdao, 266061, China \\ ${ }^{3}$ School of Geosciences and Info-Physics, Central South University, Changsha, 410083, China
}

\begin{abstract}
In recent years, the DIDSON (Dual-frequency IDentification SONar), which can provide almost-video-quality images to identify objects even in turbid water has been used in enumerating fish populations, underwater structures inspection, oil/gas leakage detection and identification, underwater security, evidence searching, ship's hulls and ports safety inspection, and underwater navigation and so on.

In this paper, a designed vessel-mounted observing systems collected DIDSON data and GPS data according to designed lines in the Dishui Lake, shanghai city. Every line area was calculated according to the depth and sonar open-angle. Trained observers counted manually each fish image in every DIDSON file. Then based on the area and number of fish observed, the average density and zoning density of the Dishui lake was calculated. At last the whole fish number and distribution was calculated based on the ARCGIS software.

The practice proved the method based on DIDSON Data is very feasible, effective and accurate for fishery resources estimating.
\end{abstract}

Keywords: DIDSON, Survey, Fishery Resources, Spatial Distribution.

\section{$1 \quad$ Introduction}

The Dishui Lake of Shanghai Lingang Town was completed on 2003. The area of whole lake is 5.56 square kilometers, the average depth is $3.7 \mathrm{~m}$, and the most deep depth is $6.2 \mathrm{~m}$. Since 2007, the management of the lake has put about 315 ton fry into the lake so far, the fry were mainly silver carp and bighead carp. At present, how much fish in the lake is a question. So the lakes management decides to detect the fishery resources for the further catching and management.

In the water, sonar equipment is the main observation and investigation equipment[1]. Acoustic fisheries resources survey is fast and efficient investigation of biological resources, and no damage for fisheries[1-3].

\footnotetext{
* Supported by the Innovation Program of Shanghai Municipal Education Commission(12ZZ159), and China Postdoctoral Science Foundation (2011M501296, 2012T50832).

** Corresponding author.
}

D. Li and Y. Chen (Eds.): CCTA 2012, Part I, IFIP AICT 392, pp. 366-375, 2013.

(C) IFIP International Federation for Information Processing 2013 
Currently, the main advanced acoustic instruments for fishery resources survey are Simrad EY-60[4], Bisonics DT-X[5], and etc.. These instruments generally utilize echo integration method[6-8] to assess the total amount of resources. When using these acoustic instruments, firstly the target strength of fish should be acquired, and then the standardized calibration should be implemented. Usually the acoustic image displayed on the screen are not dynamic, the accuracy of the fish counting is not very high. In addition, these methods also need to set the various parameters of fish, which will lead to significant differences on the identifying result[4].

This project utilized DIDSON (Dual-Frequency Identification Sonar) to detect the Dishui lake fishery resources, and acquired three days first-hand data. Based on echo counting method[9], the number of fish was counted from DIDSON image. Then the average density and zoning density of fish were calculated. At last, the total amount was calculated and the fish spatial distribution was plotted on a map based on ARCGIS software.

\section{DIDSON}

DIDSON (Dual-frequency IDentification SONar), which is invented by Washington University, can provide almost-video-quality images to identify objects even in turbid water. DIDSON has been used in fisheries management, underwater structures inspection, oil/gas leakage detection and identification, underwater security, evidence searching, ship's hulls and ports safety inspection, and underwater navigation and so on.

No other sonar in its class is able to deliver the image quality and frame rate of the DIDSON. The resulting real-time, near video-quality data is clear enough to study the behavior of darting fish, even in opaque waters.

A defacto standard for the exacting marine biology audience, this dual frequency sonar is also ideal for construction inspection, pipe laying observation, hull and berth sweeps and other mission critical jobs down to 300 meters.

The DIDSON is versatile, too, with a $1.1 \mathrm{MHz}$ detection frequency capable of ima ging targets up to 30 meters away.Stored images can be converted to AVI files and JP EGs, and a size and distance tool is included, making analysis accurate and presentati on easy. [9]
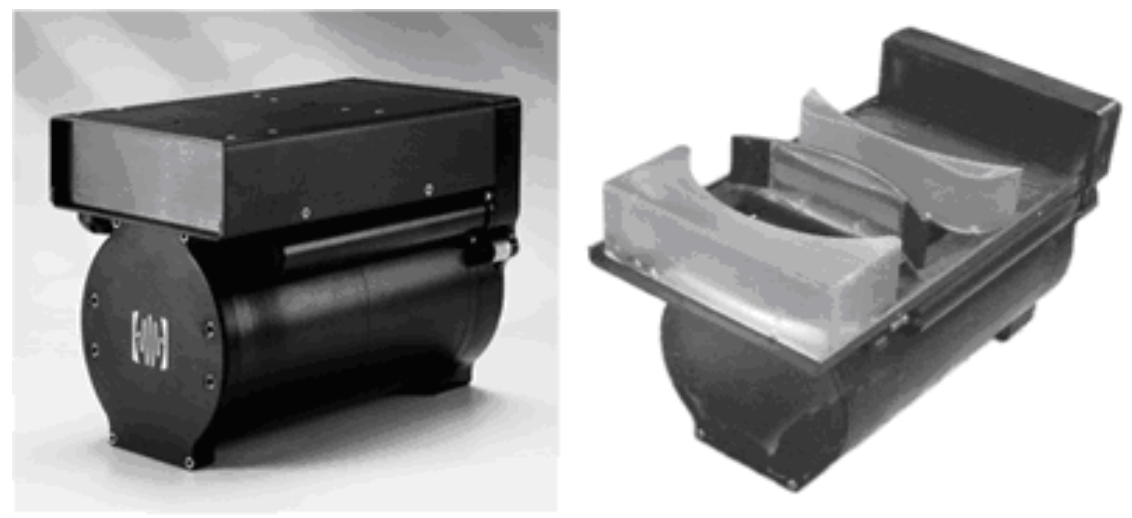

Fig. 1. Standard DIDSON (Left: main body, Right: acoustic lens ) 


\section{Fishery Resources Detection and Assessment}

\subsection{Area and Methods}

The three-day detection almost covered whole Dishui Lakes, each designed survey line spacing was $50 \mathrm{~m}$. Due to driving, weather conditions, underwater fishing nets and barriers, the actual survey routes spacing was about $100 \mathrm{~m}$ as shown in Fig. 2.

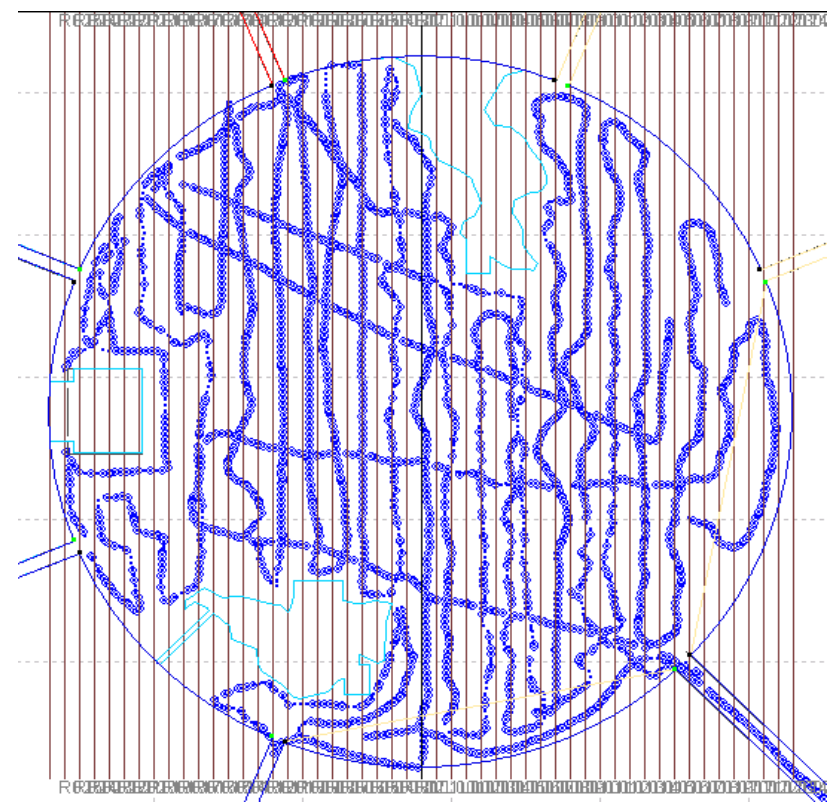

Fig. 2. The actual detecting route in Dishui lake, shanghai, China

The detection system(Fig.3) comprises the DIDSON transducer, a set-top control box, a data cable, control software, GPS and an associated laptop computer. The DIDSON transducer is directly connected to the set-top box, which is linked to the laptop via an ethernet connection. The acoustics image is transferred from the unit to the laptop via the control software, which displays the data as a streaming image. The DIDSON Image can then be directly viewed or saved on computer. The GPS positioning information is transferred into the navigation software and the actual survey routes are recorded (Fig.2). The practices improved the DIDSON transducer should be installed under water $0.5 \mathrm{~m}$ and the lens angle $45^{\circ}$ is best. 


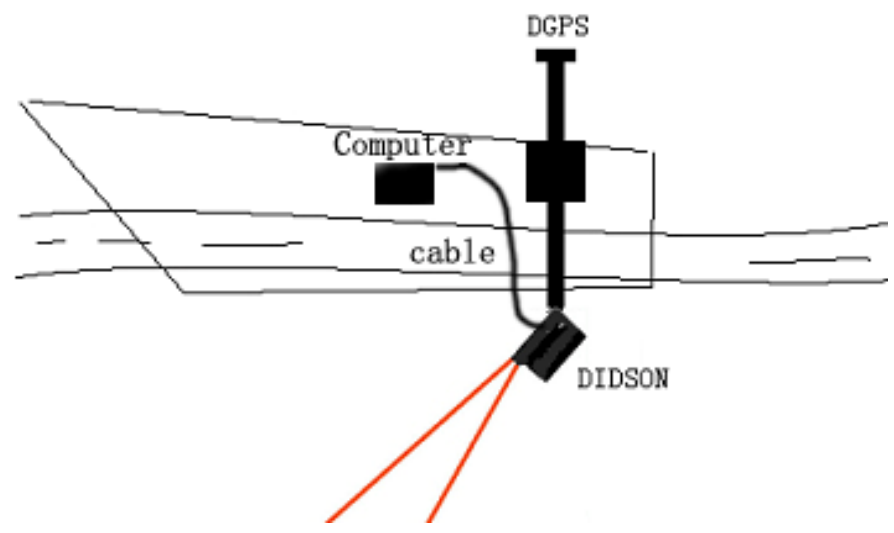

Fig. 3. Measurement of vessel and equipment installation

\subsection{Fish Counting Automatically Based on DIDSON Image}

Using DIDSON image (Fig.4) and semi-automated counting software[10,11] developed ourself, the fish number of every line was counted and the location was recorded $[12,13]$. The fish length was calculated based on the pixel value of identified fish in the image. At last, The number checked via manual review would be saved to table ( Table 1) for late statistical analysis.

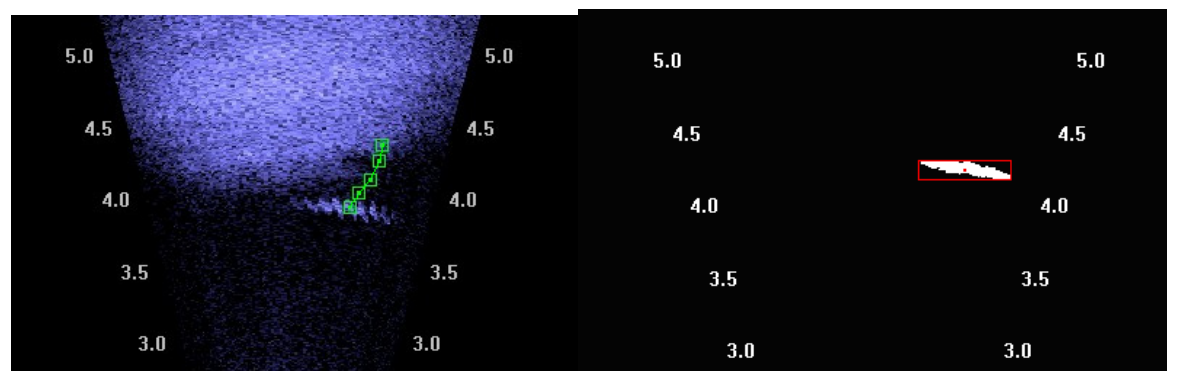

Fig. 4. Fish Identification and Tracking

Table 1. The fish count table from DIDSON image

\begin{tabular}{cccccc}
\hline $\mathrm{N}$ & $\mathrm{E}$ & Local X & Local Y & Num & Len $(\mathrm{cm})$ \\
\hline 30.90368 & 121.9308 & -36690.4516 & 44345.14614 & 1 & 96 \\
30.90346 & 121.9308 & -36714.39585 & 44347.71281 & 2 & 60 \\
30.90338 & 121.9308 & -36723.94869 & 44348.47933 & 2 & 72 \\
\hline
\end{tabular}




\subsection{Catch Statistics}

By the actual measurement data form the Dishui Lake management Company, the fish species composition, body length, weight, and other relevant data were acquired. Based on caught 41 silver carp, the relationship between weight and length (Fig.5) was established using the power function regression method.

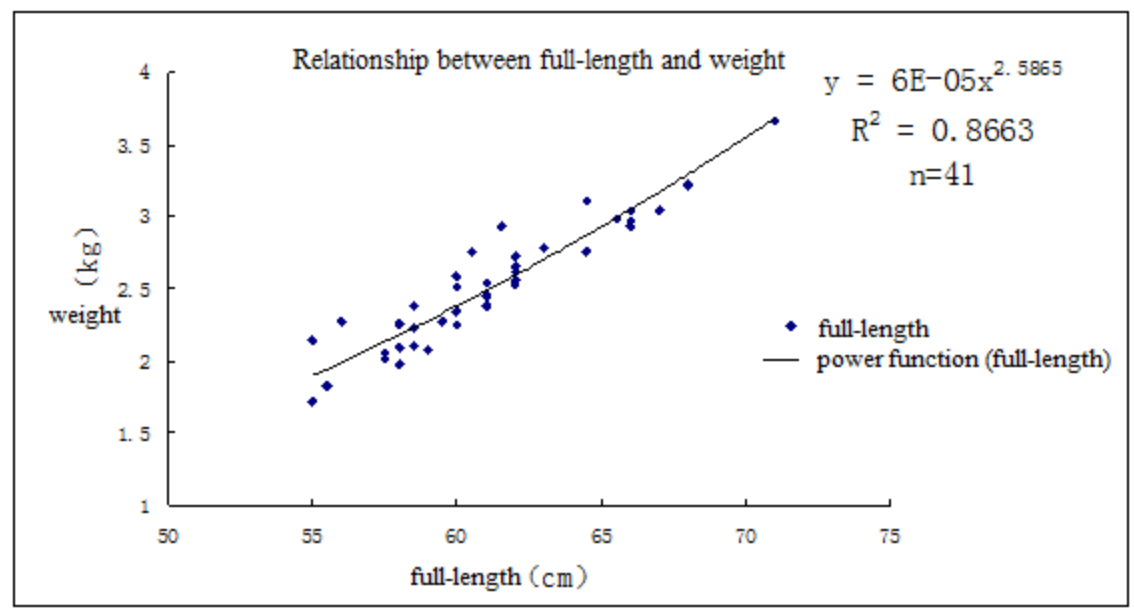

Fig. 5. Relationship between full-length and weight of silver carp ( 2 is fitting degree, $n$ is the number of samples)

The power function is

$$
\mathrm{W}=0.00006 \mathrm{~L}^{2.5865}
$$

\subsection{Fishery Resources Quantitative Assessment Methods}

The average density method and zoning density method are used in fish resources quantitative assessment in this paper.

\subsubsection{The Average Density Method}

The whole lake fish quantity value $\mathrm{N}$ is defined as

$$
N=\rho . S
$$

where $\rho$ is fish average density, $\mathrm{S}$ is the whole lake area. The value $\rho$ is calculated by every line fish density $\rho_{i}$. 


$$
\rho=\frac{1}{n} \sum_{i=1}^{n} \rho_{i}
$$

The $\rho_{i}$ is calculated by the fish number and the area of line $i$.

$$
\rho_{\mathrm{i}}=\frac{\mathrm{N}_{\mathrm{i}}}{\mathrm{S}_{\mathrm{i}}}
$$

Where the value $\mathrm{S}_{\mathrm{i}}$ is defined as

$$
\mathrm{S}_{\mathrm{i}}=\mathrm{L}_{\mathrm{i}} \times \frac{\mathrm{R}_{\mathrm{i}}}{2}=\frac{1}{2} \sum_{n=1}^{n} r_{n} l_{n}
$$

Where the $\mathrm{L}_{\mathrm{i}}$ is length of line $i$ and the $\mathrm{R}_{\mathrm{i}}$ is the slant-range from the transducer to the water bottom. The $r_{n}$ is changed by the water bottom terrain from time 1 to $\mathrm{n}$ seconds. The $l_{n}$ is computed by GPS positioning data.

Based on these methods above, the length, area, fish number, fish density of each survey line are calculated.

Table 2. The detection data of each survey line

\begin{tabular}{lllll}
\hline Line & Length $(\mathrm{m})$ & Area(m2) & fish number & $\begin{array}{l}\text { fish density } \\
\text { (num/m2) }\end{array}$ \\
\hline No1 & 3109 & 15206.1 & 485 & 0.031895095 \\
No2 & 4561 & 5697.7 & 385 & 0.067571125 \\
No3 & 1871 & 13552.6 & 1038 & 0.07659047 \\
No4 & 3740 & 12001.1 & 1152 & 0.095991201 \\
No5 & 3828 & 17333.9 & 1198 & 0.069113125 \\
No6 & 3907 & 21239.2 & 482 & 0.022693887 \\
No7 & 4605 & 3451.6 & 269 & 0.077934871 \\
No8 & 6703 & 3167.7 & 190 & 0.059980427 \\
No9 & 1827 & 22139.6 & 865 & 0.039070263 \\
No10 & 962 & 12206.9 & 823 & 0.067420885 \\
No11 & 7312 & & & \\
No12 & 3901 & & & \\
\hline
\end{tabular}

The whole lake fish density $\rho$ is 0.0608261349 via formula 3 , and the Dishui lake area is 5.56 sq.km so the whole lake fish number $N$ is 338193 . 
By the site statistics data, the mean size of Dishui lake fish is $64.26 \mathrm{~cm}$. So the fish average weight is $2.847 \mathrm{~kg}$, and the whole lake fish gross is $962835 \mathrm{~kg}$.

\subsubsection{The Zoning Density Method}

The fish average density method ignored the number difference of different regional, and it could not express the actual distribution of the fish, and it's accuracy was affected by the survey lines distribution. So the zoning density method was designed to improve the count accuracy.

The value $\mathrm{K} 1 \quad\left(\mathrm{num} / \mathrm{m}^{2}\right)$ was designed to express the fish density of every survey point.

$$
\mathrm{K} 1=\frac{\mathrm{n}}{1 \cdot \mathrm{d}}
$$

Where value $\mathrm{n}$ is the detected fish number, value 1 is the distance between two survey points, value $\mathrm{d}$ is the DIDSON scan width.

The value $\mathrm{K} 2 \quad\left(\mathrm{~kg} / \mathrm{m}^{2}\right)$ was designed to express the fish grass of every survey point.

$$
\mathrm{K} 2=\frac{\mathrm{nW}}{1 \cdot \mathrm{d}}=\frac{0.00006 \mathrm{n}}{1 \cdot \mathrm{d}} \cdot \mathrm{L}^{2.5865}
$$

Where value $\mathrm{L}(\mathrm{cm})$ is length of fish, $\mathrm{W}(\mathrm{kg})$ is the fish weight.

Based on formula $6 \& 7$, the value $\mathrm{K} 1$ and $\mathrm{K} 2$ was calculated.

Then using Inverse Distance Weighted (IDW) interpolation within ARCGIS, the whole lake fish density were processed as shown Fig.6.

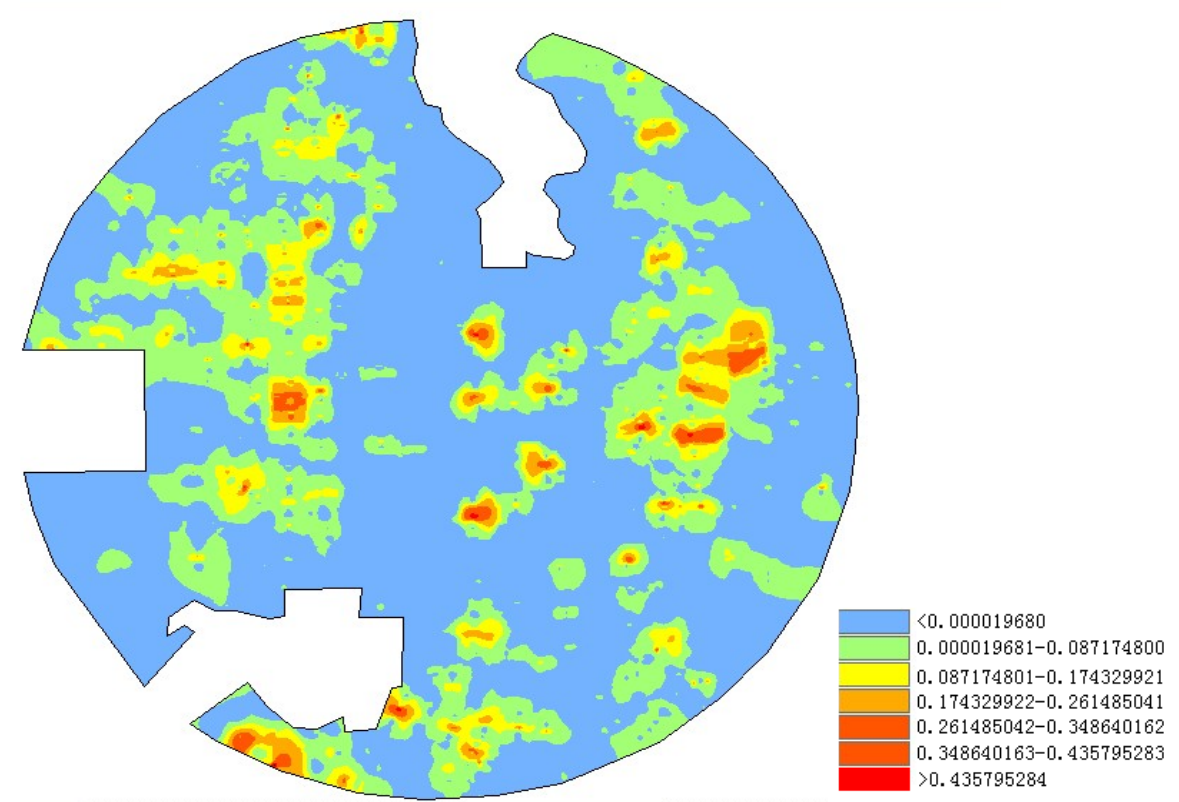

Fig. 6. Map of K1 zoning density (unit: num/m2, grid resolution 10m) 
Based on the K1 Grid data, the whole Dishui lake fish number was calculated via ARCGIS Surface Volume tool. And the whole number is 345253.

Based on the K2 Grid data, the whole Dishui lake fish gross was calculated via ARCGIS Surface Volume tool. And the whole fish gross is $939885 \mathrm{~kg}$.

\subsection{Fishery Resources Spatial Distribution}

In order to understand the fish spatial distribution of Dishui lake, the pie charts of detected fish number were plotted on a map based on the counted fish number of every points. From the map, the Dishui lake fish spatial location are expressed visually (Fig. 7). Actually the Figure 6 expresse the fish spatial distribution also.

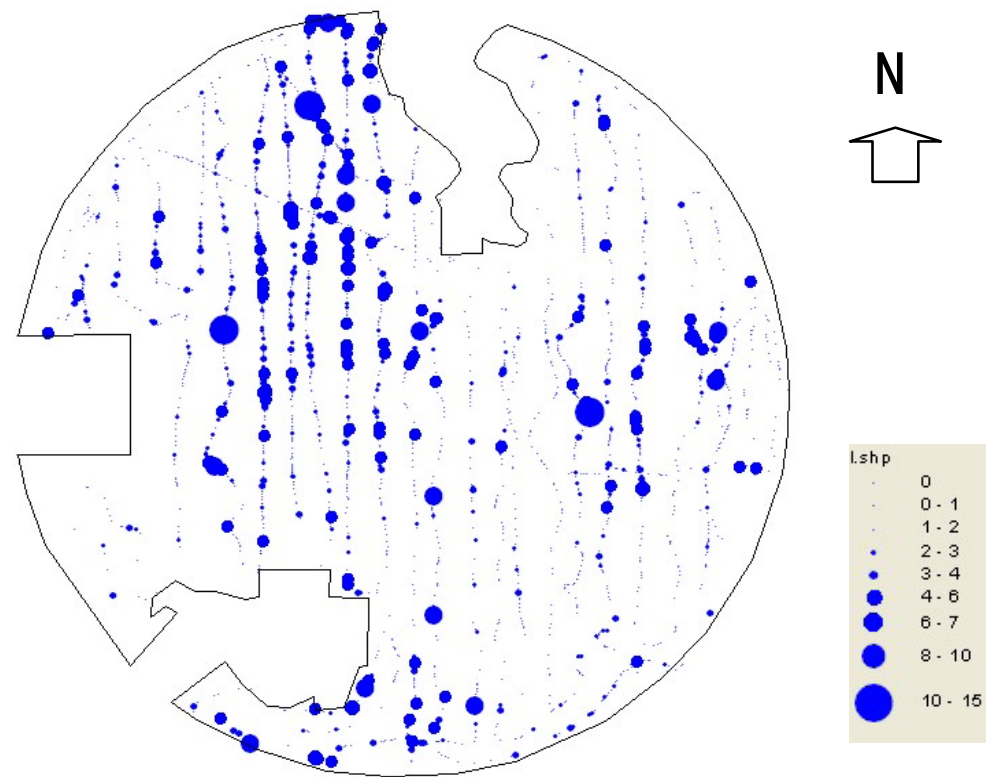

Fig. 7. Map of Dishui lake fish distribution

\section{$4 \quad$ Analysis and Discussion}

\subsection{Summary and Analysis}

In this paper, two kinds of assessment methods were used to calculate the total fish amount in Dishui lake. Whith average density method, the Dishui lake fish number is 338193 and the fish gross is $962835 \mathrm{~kg}$. And the number is 345253 via zoning density method, and the fish gross is $939885 \mathrm{~kg}$.

The assessment value by two methods is close, and the fish fry data supplied by Dishui lake management department was analyzed to prove that the two methods are 
effective. Overall, the average density method is simple, easy to operate. The zoning density method needs a large amount of calculation and requires the assist by GIS software.

Practice proved that the average method is simple and effective, can be directly applied to the assessment of fishery resources. The zoning density method witch can visually analyse fish distribution and the amount of the different regions needs lots of equally distributed detection data to interpolate the whole area data.

In comparison with the lake depth map, the fish were mainly concentrated in two deep-water areas and several estuaries. Because the main fish silver carp like warm area in winter, so they lied in deep water and lakebed. In addition, the bait was rich in estuary, so they foraged there.

\subsection{Question and Discussion}

Due to technical and capacity constraints, there are several deficiencies need for improvement.

In this detection, the fish species relatively simple and mainly concentrated in the bottom of the lake, so we used a flat density. But in many cases, different fish distributed in different water depth. So the 3D fish density in order to more accurately measure the 3D distribution will be survey in the next study.

Sonar instruments have certain blind spots, for example DIDSON has a big noise away from the lens 1 meter, where the fish can not be quantified. So how to solve this question is a key point for the next research.

When using the acoustic instruments to survey fishery resources underwater, due to the movement of the boat and the noise of boat motor, part of the fish will be driven. So how to decide the drive coefficient is very difficult but necessary, and the current research is still very scarce.

Applying DIDSON sonar to detect and assess fishery resources in China has just begun, but the practices proved that it could be used effectively in large-waters fishery resources detection and assessment.

\section{References}

1. Gunderson, D.R.: Surveys of Fisheries Resources, p. 69. John Wiley \& Sons, New York (1993)

2. Elliott, J.M., et al.: A comparison of three methods for assessing the abundance of Arctic charr, Salvelinus alpinus, in Windermere (northwest England). Fisheries Research 53(1), 39-46 (2001)

3. Tan, X., Kang, M., Tao, J., et al.: Hydroacoustic survey of fish density, spatial distribution, and behavior upstream and downstream of the Changzhou Dam on the Pearl River. China. Fish Sci., 891-901 (2011)

4. Tan, X., Shi, J., Zhang, H., et al.: Hydroacoustic assessment of fish resources in the Lake Qinghai with EY60 echosounder. Lake Sci. 21(6), 865-872 (2009) 
5. Wang, C.-R., Zhang, H., Du, H., et al.: Hydroacoustic assesment of abundance and spatial distribution of Gymnocypris przewalskii in Qinghai Lake with BioSonics DT-X echosounder. Freshwater Fisheries 41(3), 15-21 (2011)

6. Diner, N.: Correction of school geometry and density: an approach based on acoustic image simulation. ICES Coop. Res. Rep. 238, 27-51 (1999)

7. Misund, O.A., Aglen, A., Fronaes, E.: Mapping the shape, size, and density of fish schools by echo integration and a high-resolution sonar. ICES J. Sci. 52, 11-20 (1995)

8. Gauthier, S., Rose, G.A.: Acoustic observation of diel vertical migration and shoaling behaviour in Atlantic redfishes. Fish Biol. 61, 1135-1153 (2002)

9. Wang, J., Zhang, C., Wang, D.: Acoustic assessment of silver carp and bighead carp in Qinghe Reservoir. South China Fisheries Science 6(5), 50-55 (2010)

10. Han, J., Asada, A., Mizoguchi, M.: DIDSON-based Acoustic Counting Method for Juvenile Ayu Plecoglossus altivelis Migrating Upstream. Journal of the Marine Acoustics Society of Japan 36(4), 250-257 (2009)

11. Han, J., Asada, A., Honda, N., et al.: Automated Acoustic Method for Counting and Sizing Farmed Fish during Transfer using DIDSON. Fisheries Science 75, 1359-1367 (2009)

12. Tong, J., Han, J., Shen, W., Xu, P.: Mosaicing of Acoustic Video Images for Underwater Structure Inspection. In: 29th International Conference on Ocean, Offshore and Arctic Engineering, Shanghai, China (2010)

13. Tong, J.-F., Han, J., Shen, W.: Preliminary Research on the Image Processing of Acoustic Camera and Its Application in Fishery. Hunan Agricultural Sciences 17, 149-152 (2010) 